\title{
Poster PN-074
}

\section{Photodynamic therapy in neurosurgery: A proof of concesept of treatment planning system}

\author{
C. Dupont * , N. Betrouni, S. Mordon, N. Reyns, M. Vermandel
}

University Lille, INSERM, CHU Lille, U1189 - ONCO-THAI - Image Assisted Laser Therapy for Oncology, Lille, France

GlioBlastoma Multiform (GBM) is the most common primary brain tumour. 5-7 new cases for 100,000 inhabitants are recorded each year and is known for its poor prognosis: a median overall survival of 14.5 month. Standard protocol includes surgery for max-imum tumour debulking, radiation therapy and concomitant and adjuvant chemotherapy to delay tumour relapse.

Photodynamic therapy (PDT) appears as an interesting research field to improve GBM treatment. In previous studies, PDT has demonstrated promising efficacy with significantly increased overall survival. Nevertheless, PDT cannot fit into the current therapeutic modalities according to several reasons: the lack of reliable and reproducible therapy schemes (devices, light delivery system), the lack of consensus on a photosensitizer, and, more importantly, the absence of randomized and controlled multicentre clinical trial. The main objective of this study is to bring a common support for PDT planning. Here, we describe a proof of concept of treat-ment planning system (TPS) dedicated to interstitial PDT for GBM treatment.

The TPS was developed with the integrated development environment C++ Builder XE8 and the environment ArtiMED, developed in our laboratory (http://www.onco-thai.fr/ index.php? option=com content $\&$ view $=$ article $\& i d=6 \& I t e m i d=303 \& l a n g=e n)$.

This software enables stereotactic registration of DICOM images, manual segmentation of the tumour target, light sources insertion and an accelerated GPU dosimetry modelling. The dosimetry algo-rithm relying on analytical modelling was assessed. For evaluation purpose, a numerical model (partial differential equation named Helmholtz equation) was used as standard reference. This numer-ical model was implemented in the numerical modelling software COMSOL MultiPhysics 5.2 using optical coefficients provided from brain tissues infiltrated by GBM cells. Different diffusor lengths tip were modelled to evaluate irradiance at several points of the space. Relative error between reference and analytical modelling was used as evaluation metrics. For all the configuration a mean error of $13.23 \%(12.08 \%)$ was measured.

This preliminary work introduces a proof of concept of PDT planning. Although a difference appears between irradiance values obtained from numerical and from analytical model, these esti-mations can be considered as acceptable in PDT clinical context. Irradiance errors are under the geometrical precision of optical fibres localisation and the standard MRI resolution of 1 $\mathrm{mm}^{2}$. How-ever, because analytic model does not take into consideration the strong GBM heterogeneity, a Monte-Carlo method accelerated by GPU computing might be more suitable. Nevertheless, analytical model accelerated by GPU remains highly interesting for dose pre-view or optimization process when considering the development of inverse planning concept similarly to radiation therapy. Finally, this preliminary work proposes a new tool to plan interstitial or intraoperative PDT treatment and might be included in the design of future clinical trials in order to deliver PDT straightforwardly and homogenously in investigator centres. 